\title{
DEPREDACIÓN DE LAGARTIJAS POR LANIUS LUDOVICIANUS (ALCAUDÓN AMERICANO) EN EL PARQUE NACIONAL LA MALINCHE, MÉXICO.
} PREDATION OF LIZARDS BY LANIUS LUDOVICIANUS (LOGGERHEAD SHRIKE) IN LA MALINCHE NATIONAL PARK, MEXICO.

\author{
Jonathan E. Gómez-Campos ${ }^{1 *}$, Miguel A. Domínguez-Godoy ${ }^{2}$ y Aníbal H. Díaz de la Vega-PÉRez \\ ${ }^{1}$ Maestría en Ciencias Biológicas, Centro Tlaxcala de Biología de la Conducta, Universidad Autónoma de Tlaxcala. Carretera Tlaxcala-Puebla km 1.5 \\ C.P. 90000 . Tlaxcala México. \\ ${ }^{2}$ Doctorado en Ciencias Biológicas, Centro Tlaxcala de Biología de la Conducta, Universidad Autónoma de Tlaxcala. Carretera Tlaxcala-Puebla km \\ 1.5 C.P. 90000 . Tlaxcala México. \\ ${ }^{3}$ Consejo Nacional de Ciencia y Tecnología-Centro Tlaxcala de Biología de la Conducta, Universidad Autónoma de Tlaxcala. Carretera Tlaxcala- \\ Puebla km 1.5 C.P. 90000 . Tlaxcala México. \\ *Correspondence: erick.gom26@gmail.com
}

Abstract.- Here we document predation events of the Loggerhead Shrike (Lanius ludovicianus) on two species of lizards in La Malinche National Park.

Keywords.- Impalement behavior, Sceloporus aeneus, Plestiodon brevirostris.

Resumen.- En esta nota documentamos eventos de depredación del alcaudón americano (Lanius ludovicianus) sobre dos especies de lagartijas en el Parque Nacional la Malinche.

Palabras clave.- Conducta de empalamiento, Sceloporus aeneus, Plestiodon brevirostris.

El alcaudón americano (Lanius ludovicianus) es un ave con distribución amplia que se encuentra desde el sur de Canadá hasta el noroeste de Oaxaca (Miller, 1931; Vázquez et al., 2009). Se alimenta de insectos, anfibios, reptiles (Martín \& López, 1990; Yosef \& Grubb, 1993; Young et al., 2004; Cogalniceanu, 2015), aves y pequeños mamíferos (Bent, 1964; Yosef, 1996; Cogalniceanu et al., 2015). Esta ave es conocida debido a que presenta la conducta de empalamiento de sus presas, ya a que no tiene garras con la fuerza necesaria para despedazar a sus presas, por lo tanto, desarrolló un comportamiento de empalamiento para facilitar la ingesta (Cade, 1995; Cogalniceanu, 2015).

Se ha sugerido que esta conducta tiene diferentes funciones, una de ellas es una estrategia de cortejo de los machos, para exponer a sus presas en estacas naturales o artificiales como "regalos nupciales" para las hembras (Sarkozi \& Brooks, 2003), ya que a través de estas señales, las hembras pueden evaluar la calidad de los machos y su capacidad para proveer alimento (Lefranc \& Worfolk, 1997; Yosef, 2008). Otras funciones son las la de almacenar el alimento, defender un territorio y la competencia reproductiva (Smith, 1972; Cogalniceanu et al., 2015; Lara-Resendiz et al., 2019).
El 26 de junio de 2018 encontramos 12 lagartijas empaladas colocadas en estacas naturales en distintas perchas. Estas presas estaban distribuidas a lo largo de un camino de terracería entre los límites de los municipios de San Juan Ixtenco y Ziltlaltépec de Trinidad Sánchez Santos $\left(19.227744^{\circ} \mathrm{N}, 97.91145^{\circ} \mathrm{W}\right.$; WGS 84, 2600 m s.n.m.) dentro del Parque Nacional La Malinche, Tlaxcala, México. Registramos un transecto lineal de $76 \times 15 \mathrm{~m}$ a lo largo del camino rodeado de cultivos de maíz y pastizales inducidos. En total registramos 55 posibles sitos de percha de L. ludovicianus, de los cuales, únicamente siete presentaron lagartijas empaladas en estacas. La coloración de los parches ventrales y la presencia/ ausencia de escamas postcloacales, nos permitieron identificar 11 lagartijas adultas de la especie Sceloporus aeneus (seis machos y cinco hembras), así como una cría de Plestiodon brevirostris (Fig. 1). Los sitios seleccionados por L. ludovicianus para empalar a las presas fueron arbustos del género Salvia y magueyes pulqueros (Agave salmiana), de una altura de $80 \mathrm{a} 120 \mathrm{~cm}$ en los arbustos y de 140 a $180 \mathrm{~cm}$ en los magueyes aproximadamente.

Aunque hemos observado otras especies de lagartijas (Aspidoscelis costata, Barisia imbricata, Prynosoma orbiculare, Sceloporus grammicus y S. spinosus) en esta elevación dentro 


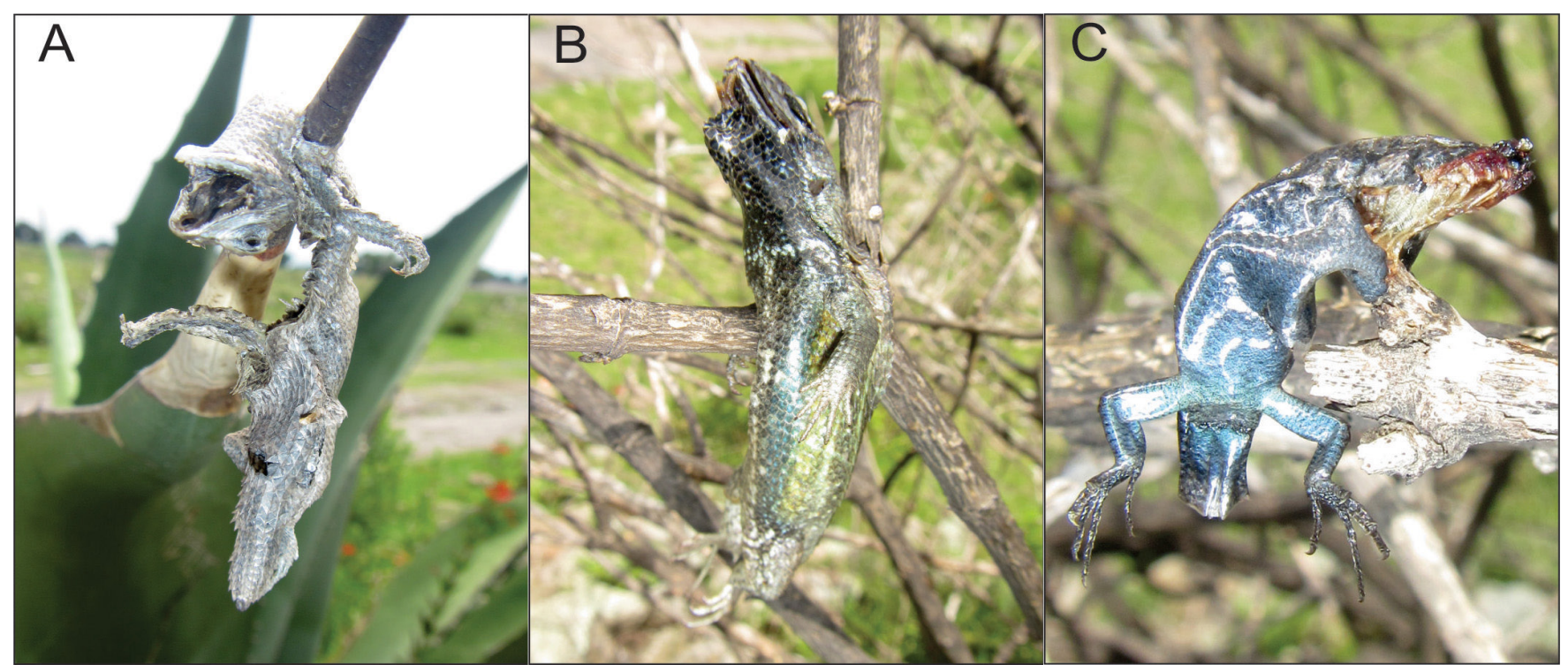

Figure 1. (A) Sceloporus aeneus in the spine of Agave salmiana, (B) Male of S. aeneus in a Salvia bush, (C) Young Plestiodon brevirostris in a Salvia bush in La Malinche National Park, Tlaxcala, Mexico. Photos: Aníbal H. Díaz de la Vega-Pérez.

Figura 1. (A) Sceloporus aeneus en espina de maguey pulquero, (B) S. aeneus macho en arbusto de Salvia, (C) Cría de Plestiodon brevirostris en arbusto de Salvia en el Parque Nacional La Malinche, Tlaxcala, México. Fotos: Aníbal H. Díaz de la Vega-Pérez.

del Parque Nacional La Malinche que podrían ser incluidas en la dieta del alcaudón americano, únicamente $S$. aeneus y $P$. brevirostris fueron registradas como presas durante este muestreo. Nuestras observaciones sugieren que $S$. aeneus es potencialmente una de las principales presas que L. ludovicianus incluye en su dieta, debido a que es muy abundante en esta elevación. Las zonas abiertas cercanas a los cultivos y pastizales inducidos podrían promover que el alcaudón americano forrajee activamente en estos sitios en el Parque Nacional La Malinche.

Agradecimientos.- Al programa de cátedras CONACYT (proyecto: 883) por el financiamiento y al personal de la Estación Científica la Malinche UATX-UNAM por el apoyo logístico.

\section{LITERATURA CITADA}

Bent, A.C. 1964. Life histories of North America wagtails, shrikes, vireos, and their allies. Dover Publication, Inc., New York.

Cade, T.J. 1995. Shrikes as predators. Proceedings of the Western Foundation of Vertebrate Zoology. 6:1-5.

Cogalniceanu, D., A. Valdeón, A. Gosá, A.A.M. Al-Hemaidi \& M.A. Castilla. 2015. Shrike predation on the lizard Mesalina adramitana in Qatar; a review of reported reptile and amphibian prey. Qscience Connect 2015:1.
Lara-Resendiz, R.A., J.H.Valdez-Villavicencio,A.G. Pérez-Delgadillo, H.D. Pinto-Santana \& P. Galina-Tessaro. 2019. Predation on flattailed horned lizard (Phrynosoma mcallii) by Loggerhead Shrike (Lanius ludovicianus). Revista Latinoamericana de Herpetología $2: 44-47$.

Lefranc, N. \& T. Worfolk. 1997. Guide to the Shrikes of the World. Yale University Press.

Martín, J. \& P. López. 1990. Amphibians and reptiles as prey of birds in Southwestern Europe. Smithsonian Herpetological information Service. 82:1-43.

Miller, A.H. 1931. Systematic revision and natural history of the American Shrikes (Lanius). University of California Publications in Zoology.

Sarkozi, D.L. \& D.M. Brooks. 2003. Eastern red bat (Lasiurus borealis) impaled by a Loggerhead Shrike (Lanius ludovicianus). Southwestern Naturalist 48:301-303.

Vázquez, L., H. Moya \& M.D.C. Arizmendi. 2009. Avifauna de la selva baja caducifolia en la cañada del río Sabino, Oaxaca, México. Revista Mexicana de Biodiversidad Vol. 80 no. 2 
Yosef, R. 1996. Loggerhead Shrike (Lanius ludovicianus). In: A. Poole and F. Gill (Eds.). The Birds of North America, No. 231. The Birds of North America, Inc., Philadelphia, PA.

Yosef, R. 2008. Familiy Laniidae. In: del Hoyo, J, A. Elliot \& DA Christie (eds). Handbook of the birds of the World. Vol. 13. Penduline Tits to Shrikes. Lynx Edicions, Barcelona, pp 732-796.
Yosef, R. \& T. Grubb. 1993. Effect of vegetation height on hunting behavior and diet of loggerhead shrikes. The Condor 95:127-131.

Young, K.V., E.D. J. Brodie \& E.D. III. Brodie. 2004. How the horned lizard got its horns. Science 304:65-65. 